\title{
Assessing vitamin status in large population surveys by measuring biomarkers and dietary intake - two case studies: folate and vitamin D
}

\author{
Christine M. Pfeiffer'*, Rosemary L. Schleicher', \\ Clifford L. Johnson ${ }^{2}$ and Paul M. Coates ${ }^{3}$
}

\footnotetext{
'National Center for Environmental Health, Centers for Disease Control and Prevention (CDC), Atlanta, GA, USA; ${ }^{2}$ National Center for Health Statistics, CDC, Hyattsville, MD, USA; ${ }^{3}$ Office of Dietary Supplements, National Institutes of Health, Bethesda, MD, USA
}

Abstract

The National Health and Nutrition Examination Survey (NHANES) provides the most comprehensive assessment of the health and nutrition status of the US population. Up-to-date reference intervals on biomarkers and dietary intake inform the scientific and public health policy communities on current status and trends over time.

The main purpose of dietary assessment methods such as the food-frequency questionnaire, food record (or diary), and 24-hr dietary recall is to estimate intake of nutrients and, together with supplement usage information, describe total intake of various foods or nutrients. As with all self-reporting methods, these tools are challenging to use and interpret. Yet, they are needed to establish dietary reference intake recommendations and to evaluate what proportion of the population meets these recommendations. While biomarkers are generally expensive and, to some degree, invasive, there is no question as to their ability to assess nutrition status. In some cases biomarkers can also be used to assess intake or function, although rarely can one biomarker fulfill all these purposes. For example, serum folate is a good indicator of folate intake, red blood cell (RBC) folate is a good status indicator, and plasma total homocysteine is a good functional indicator of one-carbon metabolism.

Using folate and vitamin D - two vitamins that are currently hotly debated in the public health arena - as two case studies, we discuss the complexities of using biomarkers and total intake information to assess nutrition status. These two examples also show how biomarkers and intake provide different information and how both are needed to evaluate and set public health policy. We also provide guidance on general requirements for using nutrition biomarkers and food and supplement intake information in longitudinal, population-based surveys.

Keywords: nutrition survey; NHANES; monitoring; trend; biochemical indicator; nutrition status; food intake; dietary questionnaire; folate; vitamin $D$

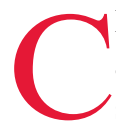
linical research studies, randomized clinical trials, and population-based surveys or surveillance systems provide valuable information to research as well as public health policy community in their ongoing discussion on the importance of vitamins in health outcomes. Public health policy and intervention programs rely on these sources of information. One critical set of surveillance information needed is the nutrition status of the population, at present and in the past. The National Health and Nutrition Examination Survey (NHANES) is planned and conducted by the National Center for Health Statistics (NCHS), Centers for Disease Control and Prevention (CDC), and its primary objective is to assess the health and nutrition status of adults and children in the United States (1). NHANES is the most significant populationbased survey to provide questionnaire, examination, and laboratory data to the scientific and public health policy 
community on a variety of nutrition and health topics. This US survey has evolved for the past 50 years, first as targeted health surveys in the 1960s and then as periodic surveys of health and nutrition from the early 1970s to the middle 1990s. Since 1999, NHANES has been a continuous survey conducted in 2-year cycles, covering the entire age range of the civilian, non-institutionalized US population. NHANES is unique in that it combines home interviews with health tests performed in a Mobile Examination Center (MEC), where biologic specimens are also collected for biochemical analyses.

Measuring biomarkers and assessing food and supplement intake are the two main tools used to assess the vitamin status of a population. Because each tool has advantages and disadvantages, frequently they are performed together for better interpretation. Aside from evaluating inadequate or excess vitamin intake levels and the relation between vitamin status and health outcomes, this information can potentially assist in the following public health activities:

1. Establishing and improving existing population reference intervals that physicians, clinicians, nutritionists, scientists, and public health officials can use to determine whether a person or a group of people has an unusually high or low level of a biochemical indicator or intake.

2. Determining whether the nutrition status of special population groups such as minorities, children, women of childbearing age, or the elderly is different from that of other groups or whether it needs improvement.

3. Tracking trends in the nutrition status of the population.

4. Assessing the effectiveness of public health efforts to improve the nutrition status of the population.

This article discusses the complexities of using biomarkers and total intake information to assess nutrition status and set and evaluate public health policy by using folate and vitamin D as two case studies.

\section{Methods and materials}

\section{Biochemical assessment to evaluate vitamin status in NHANES}

The main purpose of biochemical measurements is to assess nutrition status generally as a sum of food intake, supplement use, bioavailability, metabolism, and excretion. While field tests are available for some clinical parameters such as hemoglobin or glucose, nutrition biomarkers generally must be analyzed in the laboratory using more complex biochemical assays based on analytical principles such as chromatography, immunoassay, or colorimetry. Because biomarkers are not self-reported, they typically correctly reflect what we believe to be the nutrition status. Although biomarkers can also be used to directly assess intake or function, rarely can one biomarker fulfill all of these purposes. Panels of biomarkers are sometimes used for enhanced interpretation. Limitations of biomarkers include expensive procedures and equipment and usually invasive sampling. To obtain reliable results, laboratory methods must undergo thorough validation and their quality must be continuously assessed. One less-discussed concern is that biochemical methods to assess nutrition status have generally not been standardized, which leads to problems with poorly comparable data among different assays and laboratories.

In NHANES, blood is drawn and urine is collected in the MEC; aliquots of these specimens are processed in a controlled environment following standardized protocols. Specimens are stored at $-20^{\circ} \mathrm{C}$ at the MEC until the end of the week when they are shipped on dry ice to the testing laboratories. Early attempts to use multiple laboratories per test for the nutrition biomarkers in the Ten State Nutrition Survey (1968-1969) to allow for fast sample turn-around resulted in problems of non-comparability of data. A central laboratory using reference analytical methods was therefore established in 1970 at the CDC for HANES I (1971-1975) (2). This allowed for consistent, coordinated, and high-quality biomarker measurements. The concept of a central laboratory - at least for medium and high complexity testing - is still in place today.

The 'National Report on Biochemical Indicators of Diet and Nutrition in the US Population 1999-2002' is the most comprehensive and current source of reference interval tables for 27 nutrition biomarkers in the US population (3). The CDC's National Center for Environmental Health $(\mathrm{NCEH})$, plans to release periodic updates of this report. The next report, scheduled to be released in 2012, will cover reference data for $>50$ nutrition biomarkers from NHANES 2003-2006.

\section{Dietary assessment to evaluate vitamin status in large population surveys}

The purpose of dietary assessment methods is to evaluate intake of foods or nutrients and, combined with supplement usage information, describe total intake of various nutrients. The main assessment methods are food frequency questionnaire (FFQ), 24-hr dietary recall and the food record (or diary). All methods have strengths and weaknesses; the method selected should be dependent on the research question. These methods are relatively easy and inexpensive (with the possible exception of the 24-hr recall) to conduct, and they have the advantage of being non-invasive. Technologic advances in tools (interviewer, computer, Internet, cell phone) as well as data processing (manual, computer, specialized 
software, personal digital assistants) have facilitated and standardized the administration of these methods (4). Three main FFQs are used - Block, Willett, and the National Cancer Institute (NCI) - and a comparative validation has been conducted (5). The two commonly used 24-hr recall interviewing systems are the University of Minnesota's Nutrition Data System for Research (6) and the USDA's Automated Multi-Pass Method (7). The $\mathrm{NCI}$ is developing a computer-based, 24-hr recall interviewing system that does not require specially trained interviewers. However, as with all self-reporting methods, these tools are challenging to use and interpret. The process of recording intake influences diet and introduces bias. Under-reporting is common, particularly for obese persons (8) and can alter relationships with both biomarkers and health outcomes. Typically, correlation coefficients between nutrient intake and the biomarker value are $<0.5(9,10)$. Yet, even with these shortcomings, information gained from dietary assessment methods is vital in establishing guidelines for dietary reference intakes and evaluating what proportion of the population meets these recommendations and who the potential groups of concern are.

Since 2002, NHANES collects two 24-hr dietary recalls for every participant using the USDA's Automated Multi-Pass Method. The first 24-hr recall is conducted at the MEC; the second is conducted by telephone 3-10 days later. Also since 2002, NHANES uses either small, targeted FFQs or an NCI-developed FFQ (11). The NHANES FFQ, formerly called Food Propensity Questionnaire, is mailed to all participants aged 2 and older who completed at least one $24-\mathrm{hr}$ recall. It collects much of the same information as the Block or Willet FFQ but does not ask about portion size, thereby minimizing respondent burden. As suggested in the NHANES Dietary Tutorial (12), the FFQ data were not intended to be used directly for nutrient intake estimation, but rather as covariates when estimating usual dietary intakes from the 24-hr recalls. Generally, day-to-day variation in intake for an individual is greater than person-to-person variation within a population. To avoid this excessive intra-individual variation, which can lead to serious problems in data interpretation, NCI has developed a statistical modeling method to estimate usual dietary intakes representing a more accurate average daily intake of a nutrient or food and thereby being more reflective of long-term exposure (13). It requires the availability of data from one or more non-consecutive, 24-hr recalls or food records for a representative sample of persons from the population of interest.

'What We Eat in America' (WWEIA) is a report containing data from the dietary intake interview component of NHANES (14). It is an integration of two nationwide surveys, the US Department of Agriculture's (USDA) Continuing Survey of Food Intakes by
Individuals (CSFII) and NCHS's NHANES. The two surveys were integrated in 2002. NCHS is responsible for sample design and data collection; USDA is responsible for dietary data-collection methods, maintenance of the databases used to code and process the data, and data review and processing. The USDA Food and Nutrient Databases for Dietary Studies (FNDDS) is a resource that is used to code dietary intakes and to calculate nutrients for WWEIA (15). The FNDDS is based on nutrient values in the USDA National Nutrient Database for Standard Reference, the most recent release of which is SR23 (16). For each NHANES 2-year data release cycle, WWEIA generates two dietary intake data files. The individual foods file contains one record per food for each survey participant for each 24-hr dietary recall. It provides information on the estimated nutrient consumption, when and where the food was eaten, and the amount of food eaten. The total nutrient intakes file contains one record per day for each survey participant for each 24-hr dietary recall. It provides information on daily totals of food energy and nutrient intakes, daily intake of water, and whether the intake was typical for the participant.

To assess supplement usage in NHANES, the interviewer completes a household dietary supplement questionnaire with information provided by the participant. This questionnaire captures dietary supplement use over the past 30 days, listing the name and type of the supplement, frequency of use, duration of use, and amount taken. Since 2007, during the MEC visit, the participant has been asked about supplement use on the previous day; this information can be matched with the 24-hr dietary recall. An average daily intake of dietary supplements can be calculated from the information collected at the home of the participant.

\section{Results and discussion}

\section{Case study one - assessment of folate status in NHANES}

In 1996, the FDA mandated that enriched grain products be fortified with folic acid $(140 \mu \mathrm{g}$ per $100 \mathrm{~g})$ to reduce the risk of neural-tube defects (NTD) in newborns (17). This regulation became effective January 1998. Folic acid fortification provides an excellent account of the various pieces of information that were needed to implement such a public health policy (18). To establish the need for folic acid fortification, a determination had to be made that: (1) the total folate intake (foods and supplements) was low, (2) the prevalence of folate deficiency as assessed through biomarkers was high, (3) a significant number of folate-preventable NTDs were present, and (4) alternative strategies to increase folate intake were not promising. Benefits and risks of fortification had to be weighed. The folic acid fortification amount had to be determined through modeling by identifying target 
foods for fortification, assessing the frequency and amounts of the target foods consumed by various population groups, and using dose-response information from intervention trials to estimate the expected increase in biologic response.

NHANES has monitored folate status through biochemical measurements and dietary intake assessment both pre- and post-fortification and has therefore been an excellent tool to assess the impact of the US folic acid fortification program.

\section{Biochemical assessment of folate status}

Serum folate, red blood cell (RBC) folate, and plasma total homocysteine (tHcy) are the three most commonly used biochemical indicators to assess folate status (19). Serum folate is indicative of recent folate intake and short-term status, while RBC folate better indicates folate stores and long-term status. Plasma tHcy is responsive to changes in folate intake, but it is mainly considered a good functional indicator of B-vitamin status; plasma tHcy is elevated as a result of low folate, vitamin B2, B6, and/or B12 levels.

Measurements of serum and RBC folate levels among a full set of participants were part of NHANES III (1988-1994) as well as of each survey cycle since the introduction of the continuous NHANES survey in 1999 (20). The same BioRad Quanta Phase II radioassay was used from 1991 to 2006 for both matrices. An earlier version of the assay, the Quanta Phase I radioassay, was used from 1988 to 1991; however, the data were adjusted for the method differences between the two assays prior to release (21).

Measurements of plasma tHcy levels by the Abbott fluorescence polarization immunoassay among a full set of participants were part of NHANES 1999-2004 (22). Because optimally prepared EDTA plasma was not available during NHANES III, tHcy levels were analyzed by HPLC in surplus serum from phase II of NHANES III (1991-1994) (23). Method-comparison studies to assess the change in matrix and methods were conducted and discussed elsewhere $(22,24)$.

Before implementing folate fortification, FDA intake modeling estimated that consumers with low folate intake would increase their folate intake by $80-100 \mu \mathrm{g}$ per day (25). Short-term folic acid supplementation trials showed that serum folate levels increased by approximately $20-30 \%$ in response to a $100 \mu \mathrm{g}$ per day increase in folic acid intake (26). No information was available on the magnitude of changes in serum folate levels as a result of continuous daily exposure to folic acid from fortified food products. By applying the same laboratory method (BioRad radioassay) - held to strict quality assurance requirements to ensure comparability of data over time to post-fortification blood samples as had been used with pre-fortification samples, we showed that folate status has greatly improved in the entire US population as well as in the target group of women of childbearing age (27). Serum folate levels stabilized between 1999 and 2006 (20) (Fig. 1). The increase in serum folate levels from pre- to post-fortification was however, much larger than expected from short-term folic acid supplementation trials - they nearly tripled. This demonstrates the value of biomonitoring; it reflects the net effect of many uncertainties surrounding public health decisions such as fortification (i.e. under-reporting of foods consumed, increased consumer selection of folate-rich foods because of health claims, and increasing availability of the numbers and types of folate-fortified, non-standardized foods).

Short-term folic acid supplementation trials showed that $\mathrm{RBC}$ folate levels increased by $\sim 10 \%$ in response to a $100 \mu \mathrm{g}$ per day increase in folic acid intake (28). In pre- to post-fortification NHANES, RBC folate levels increased much more than expected from short-term folic acid supplementation trials - they nearly doubled (27). The prevalence of low RBC folate levels in women of childbearing age decreased from $\sim 40 \%$ pre-fortification to $\sim 5 \%$ post-fortification and stabilized between 1999 and 2006 (19) (Fig. 2).

As would be expected from the inverse relationship between folate and tHcy, elevated tHcy levels have decreased from pre- to post-fortification in older men ( $\geq 60$ years) from 30 to $15 \%$ and in older women from 20 to $10 \%(22)$.

In 2007, the manufacturer discontinued the BioRad folate radioassay. Because continued monitoring of folate status through NHANES was still necessary, the traditional microbiologic growth assay (MA) using Lactobacillus casei - used by some countries in their national nutrition surveys for population monitoring - was selected to measure folate levels in serum and RBCs in the US population for 2007 and thereafter. More recently, after input from a 2010 roundtable of folate experts, CDC decided that liquid chromatography coupled to

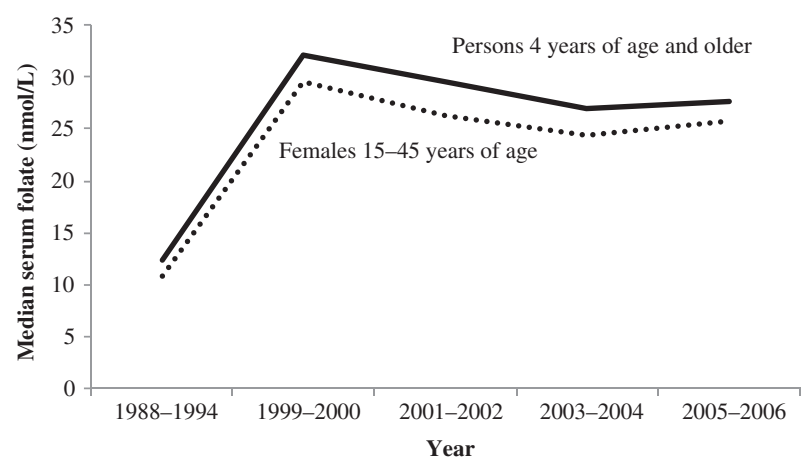

Fig. 1. Serum folate levels in the US population and women of childbearing age before (1988-1994) and after folic acid fortification (1999-2006), NHANES 1988-2006. Source: Data obtained from ref (20). 


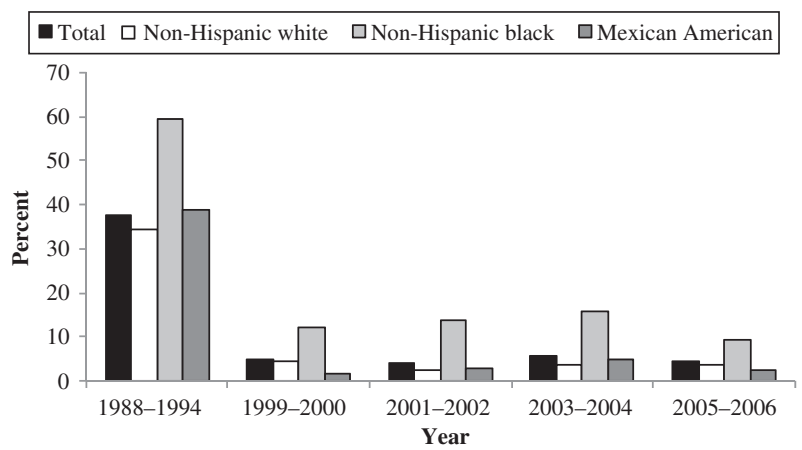

Fig. 2. Prevalence of low red blood cell folate levels $(<317$ nmol/L) in US women of childbearing age before (19881994) and after folic acid fortification (1999-2006), NHANES 1988-2006. Source: Data obtained from ref (20).

tandem mass spectrometry (LC-MS/MS) should be used to continue monitoring serum folate for NHANES 2011 and thereafter. This assay measures individual folate forms, in particular, unmetabolized folic acid, which is under investigation for potential health effects because it is not a physiological form of folate.

Total intake assessment of folate status

The various sources of folate intake are foods (which contain mainly reduced folate forms), fortified foods such as enriched grain products and ready-to-eat cereals (which contain added folic acid in addition to natural reduced folate forms), and dietary supplements (which contain folic acid only). Due to the increased bioavailability of folic acid compared with food folate, one dietary folate equivalent (DFE) has been defined as equivalent to $1 \mu \mathrm{g}$ of food folate, $0.6 \mu \mathrm{g}$ of folic acid from fortified food, $0.6 \mu \mathrm{g}$ of folic acid from a supplement taken with food, or $0.5 \mu \mathrm{g}$ of folic acid on an empty stomach (19).

The recommended dietary allowance (RDA) of DFEs for adult men and women is $400 \mu \mathrm{g}$ per day, while the estimated average requirement (EAR) of DFEs is $320 \mu \mathrm{g}$ per day (18). The tolerable upper intake level (UL) for adults is set at $1,000 \mu \mathrm{g}$ per day of folate from fortified food or as a supplement (i.e. folic acid), exclusive of food folate (19). To reduce the risk of NTDs among newborns, the Public Health Service recommends that women capable of becoming pregnant take $400 \mu \mathrm{g}$ of folic acid daily from fortified foods, supplements, or both, in addition to consuming food folate from a varied diet (29).

A study was conducted (30) to assess changes in folate total intake from pre- (1988-1994) to post-fortification (1999-2000) in NHANES. The study found that after fortification, the category 'bread, rolls, and crackers' became the single largest contributor of total folate to the American diet (15.6\% of total intake), surpassing vegetables, which were the number one folate food source before fortification. The population's mean total folate intake increased by $28 \%$ from $275 \mu \mathrm{g}$ to $351 \mu \mathrm{g}$ per day. However, at the time, the dietary folate data in the NHANES nutrient database were reported as total folate intake (food folate + folic acid from fortification) in micrograms per day not as DFE and intake was, therefore, underestimated.

Another report (31) assessed post-fortification (20012002) total folic acid intake in women of childbearing age. The study found that no race/ethnic group consumed the $400 \mu \mathrm{g}$ of folic acid daily from fortified food, supplements, or both as recommended by the Public Health Service for women capable of becoming pregnant (29). The average estimated daily total folic acid intake was $221 \mu \mathrm{g}$; however, this represents an underestimation of $\sim 10 \%$ because a second $24-\mathrm{hr}$ dietary recall was not available in NHANES 2001-2002 to calculate usual intakes.

A study was conducted (32) to assess post-fortification (2003-2006) total folate and folic acid intake in the entire population in a data set where two 24-hr dietary recalls were available and usual intakes were calculated. While the average total folate intakes (diet + supplements) for various age groups of adults 19 years and older were quite high (645-900 DFE for women and 774-938 DFE for men), $13-17 \%$ of women and $3.6-6.3 \%$ of men had a folate intake below the EAR. A large portion of the total folate intake was provided by diet only (including fortified foods): 454-470 DFE for women and 558653 DFE for men. The average total folic acid intakes (diet+supplements) were 274-407 $\mu \mathrm{g}$ for women and $317-421 \mu \mathrm{g}$ for men; $2.2-5.2 \%$ of women and $2.1-5.0 \%$ of men exceeded the UL for adults.

A recent report (33) assessed the post-fortification (2003-2006) source of folic acid intake and the proportion of the entire population that exceeded the UL. The study found that $2.7 \%$ of the total US population exceeded the UL for folic acid. Some participants exceeded the UL, but only if the source of folic acid was supplements in addition to enriched grain products and ready-to-eat cereals (5.5 and 9.4\%, respectively). Of the supplement users, $\sim 1 \%$ exceeded the UL if the supplement amount was $\leq 400 \mu \mathrm{g}$ per day, while $48 \%$ exceeded the UL if the amount of supplement was $>400$ $\mu \mathrm{g}$ per day.

Summary of biochemical and dietary assessment of folate status

The NHANES blood-biomarker levels have responded as expected - serum and RBC folate have increased and plasma tHcy has decreased - but the magnitude of change was larger than anticipated from small-scale intervention studies. Dietary intake levels of folic acid and total folate have also responded as expected - they have increased in response to folic acid fortification. However, folate intake is still below the EAR for a small portion of 
the population, and women of childbearing age still do not consume, on average, the recommended $400 \mu \mathrm{g}$ of folic acid daily. Only a small fraction of adults who consume supplements exceed the UL of folic acid. One issue in examining the relationship between biomarker data and dietary assessment data is that they sometimes reflect differential time periods. For example, we adjust the nutrient intakes reported in the 24-hr recall to reflect long-term exposure and, therefore, they may be more comparable to status assessed by $\mathrm{RBC}$ folate than by serum folate. The folate case study also shows how each data source, biomarkers, and questionnaires, provides valuable information on its own. Public health officials need both types of information - in addition to knowing how NTD rates have changed over time to judge whether fortification has achieved the desired impact in the group of interest without exposing other population groups to excessive amounts of folic acid. Biomarker levels of folate status are associated with a number of health outcomes beyond NTDs - from anemia to various chronic disease conditions - that are currently under investigation. The ability to integrate intake information with biomarker information in a large population survey such as NHANES opens the door to exploratory research and hypothesis generation for future targeted nutrition and health studies.

\section{Case study two - assessment of vitamin D status in NHANES}

Vitamin D is one of the most talked about nutrients today in the United States as well as worldwide. In recent years, low vitamin D blood levels have been associated with increased risk for numerous health outcomes beyond risk for rickets in children and osteomalacia in adults. Some of these health outcomes include various cancers, cardiovascular disease, autoimmune conditions (such as multiple sclerosis), dementia, diabetes, and glucose intolerance (34). At the same time, excessive amounts of vitamin $\mathrm{D}$ in blood can become toxic and produce a variety of health problems (35). Therefore, both excessive and deficient levels of vitamin D are important. While there is no unanimity with respect to vitamin D and various health outcomes, we have an obvious need to know the vitamin D status of the US population and whether it has changed over time. It is also important to know how much of the population's diet is contributing to vitamin D status, what foods provide the most vitamin $\mathrm{D}$ to the population, and whether dietary supplements provide a significant amount of vitamin $\mathrm{D}$ to the population taking them. Finally, the scientific and public policy community need to know how much vitamin D we need and how much is too much. NHANES has been providing answers to some of these questions since NHANES III, although much needed improvements in the USDA National Nutrient Database for Standard
Reference of foods containing vitamin D (36) were only recently made available with SR22. NHANES does not provide direct information about participants' sun exposure, which catalyzes cutaneous synthesis of vitamin D. As endogenously synthesized vitamin D3 is a significant source for most persons, this unusual information gap makes biomarker measurements even more important to assess the vitamin D status of the US population.

Biochemical assessment of vitamin $D$ status

Serum 25-hydroxyvitamin D $(25(\mathrm{OH}) \mathrm{D})$ levels are generally considered the best indicator of vitamin D status (35). The DiaSorin radioassay was used to assess 25(OH)D in NHANES III (1988-1994) and in the continuous NHANES starting in 2000 through the 2005-2006 survey cycle (37). However, the manufacturer introduced an assay reformulation in the late 1990s that required us to bridge the gap between data obtained by using two versions of the assay to allow the interpretation of trends over time (37). Specifically, the change from the original to the reformulated assay produced $25(\mathrm{OH}) \mathrm{D}$ results that were on average $12 \%$ lower (38). The observed age-standardized 25(OH)D means were $10-18 \mathrm{nmol} / \mathrm{L}$ higher in NHANES III (black bars), in
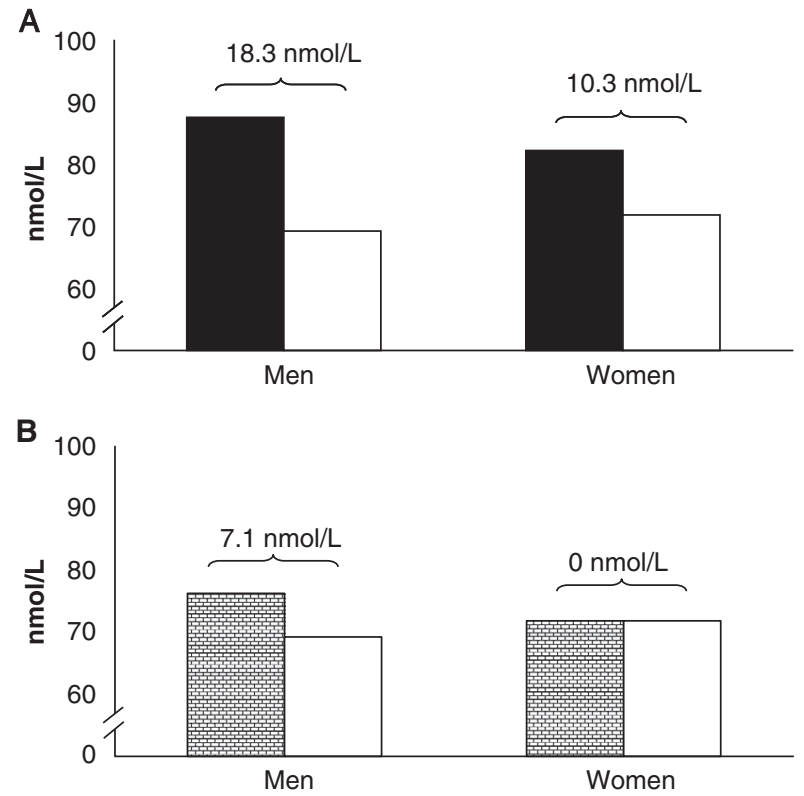

Fig. 3. Serum 25-hydroxyvitamin D levels in non-Hispanic whites, aged 20-50 years, examined April-October during NHANES III (1988-1994) and NHANES 2003-2004. Panel A: Observed age-standardized means for NHANES III (black bars) and NHANES 2003-2004 (white bars). Panel B: Predicted age-standardized means for NHANES III (brick-patterned bars), assuming that the reformulated radioassay was used, compared with observed means from NHANES 2003-2004 (white bars). Source: Data obtained from ref (38). 
which the original DiaSorin assay was used, than in NHANES 2003-2004 (white bars), in which the reformulated assay was used (Fig. 3, Panel A). When the NHANES III values were adjusted for the change in assay, the difference in age-standardized means between surveys was reduced by $10.3-11.2 \mathrm{nmol} / \mathrm{L}$, depending on sex (Fig. 3, Panel B). This means that a large portion of the previous difference between these two surveys was attributable to changes from the original to the reformulated assay and not to the real difference in population levels of $25(\mathrm{OH}) \mathrm{D}$. A smaller portion of the previous difference between these two surveys was attributable to altered behavior, specifically an increase in body mass index, decrease in sun exposure, or decrease in milk consumption (data not shown) (38).

Due to some shortcomings of the immunoassay methodology for measuring $25(\mathrm{OH}) \mathrm{D}$, after input from a roundtable of vitamin D experts, CDC decided that LCMS/MS should be used for NHANES 2007 and thereafter (37). This assay measures the individual $25(\mathrm{OH}) \mathrm{D}$ forms and has been standardized to the higher-order reference methodology developed by the National Institute of Standards and Technology.

A second important issue with vitamin $\mathrm{D}$ is that the experts disagree on the most appropriate $25(\mathrm{OH}) \mathrm{D}$ cutpoint for optimal health (39). Since 1997, the cutpoint of $<27.5 \mathrm{nmol} / \mathrm{L}$ has been used in the United States to define deficiency status for vitamin D (35). This cutpoint was associated with increased risk of rickets and osteomalacia. In recent years, other cutpoints (i.e. $50 \mathrm{nmol} / \mathrm{L}$ and $80 \mathrm{nmol} / \mathrm{L}$ ) have been proposed by members of the research community as more appropriate for 'inadequate' or 'at risk' levels, especially for other public health concerns or diseases. The 2010 IOM report (40) reevaluated the evidence for cutpoints and concluded that $25(\mathrm{OH}) \mathrm{D}<30 \mathrm{nmol} / \mathrm{L}$ and $30-50 \mathrm{nmol} / \mathrm{L}$ places individuals at risk for deficiency and inadequacy, respectively.

The prevalence of deficiency at the cutpoint of 27.5 $\mathrm{nmol} / \mathrm{L}$, a value that is close to $30 \mathrm{nmol} / \mathrm{L}$, is $<6 \%$ for all

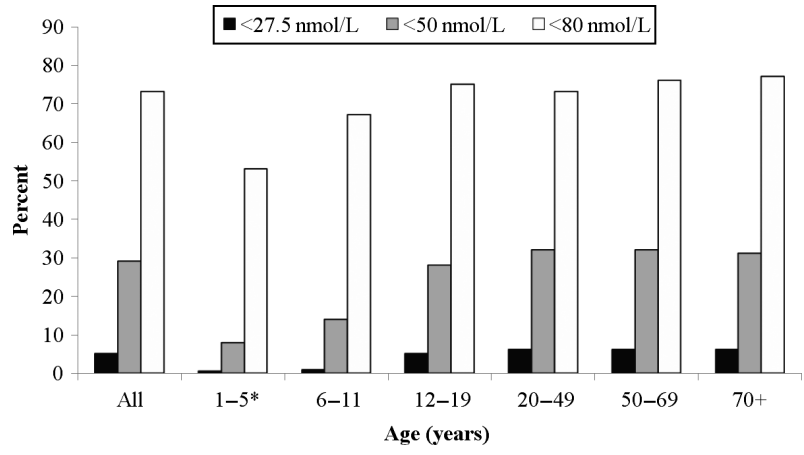

Fig. 4. Prevalence of low serum 25-hydroxyvitamin D levels in the US population by cutoff, NHANES 2000-2004. *Data for ages 1-5 years are available from NHANES 2003-2004 only. Source: Data obtained from ref (39). population age groups in NHANES 2000-2004 (Fig. 4) (39). The cutpoint of $50 \mathrm{nmol} / \mathrm{L}$ produces combined prevalences of vitamin D deficiency and inadequacy of $\sim 30 \%$ for the various population age groups. Differences among race/ethnic groups in the prevalence of low levels of serum $25(\mathrm{OH}) \mathrm{D}$ have also been found, but the meaning of these differences is unclear (39). African Americans have reduced rates of fracture and osteoporosis compared with Whites despite reduced cutaneous synthesis of vitamin D and lower 25(OH)D levels throughout life (40).

Total intake assessment of vitamin $D$ status

With respect to vitamin D intake from foods and dietary supplements, the primary questions are: 'Is intake adequate?' and 'What are the groups of concern?' Based on the 2010 IOM report (40), the new RDA for vitamin $\mathrm{D}$ is 600 international units (IU) for those between 1 and 70 years of age. The RDA for those $>70$ years is $800 \mathrm{IU}$. The EAR for vitamin D is $400 \mathrm{IU}$ per day for persons 1 year and older and adequate intake (AI) is $400 \mathrm{IU}$ per day for infants. For pregnant and lactating women, the EAR is $400 \mathrm{IU}$ per day. The UL for those 9 years and older is 4,000 IU per day, those 4-8 years 3,000 IU per day, those 1-3 years 2,500 IU per day, those 7-12 months 2,500 IU per day, and those $0-6$ months $1,000 \mathrm{IU}$ per day.

Based on an analysis of vitamin D intake data from NHANES 2005-2006 (foods alone and foods plus dietary supplements) using the 1997 IOM guidelines, much of the adult US population is not meeting the recommended adequate intake for vitamin D (40). This is especially the case for men $>50$ years (Fig. 5). In those age groups, only $24-36 \%$ meet the old AI recommendations and dietary supplements are a significant source of vitamin D intake, accounting for $\sim 80 \%$ of the intake in those $51-70$ years and nearly all of the intake in those older than 70 years. For females, with the exception of 14- to 18 -year-old women, the pattern for total intake is similar

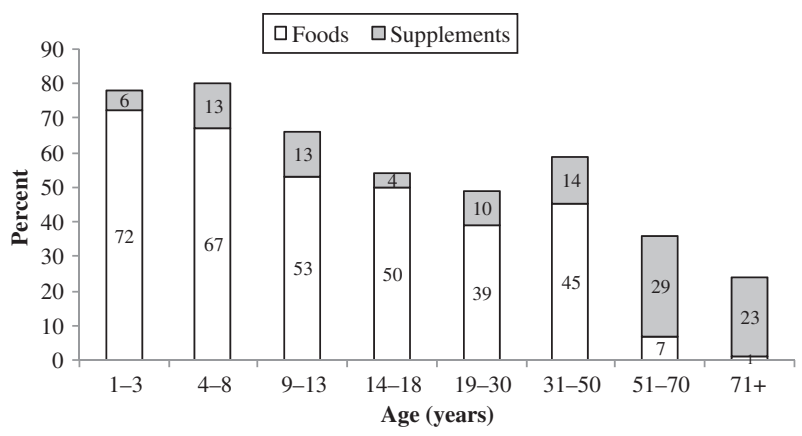

Fig. 5. Percentage of US males with vitamin D intakes from foods and dietary supplements above the adequate intake (AI), NHANES 2005-2006. Source: Data obtained from ref (41). 
to that for males (data not shown) (40). Fewer than one-third of women 14-18 years and $\geq 71$ years have adequate intakes based on the older guidelines. For women aged $\geq 14$ years, dietary supplements contribute significantly to total vitamin D intake. Comparing the $1997 \mathrm{AI}$ and 2010 EAR recommendations as they apply to populations, recommendations have doubled for all groups except those 51-70 years for whom the recommendation is the same (400 IU for AI or EAR) and those $>70$ years for whom the recommendation has decreased (1997: 600 IU AI vs 2010: 400 IU EAR). Thus, the percentage of persons meeting the new intake recommendations are expected to be lower in younger age groups and higher in those $>70$ years. However, when setting these recommendations, the IOM pointed out that reported intakes are expected to be underestimations of actual intakes (40).

Summary of biochemical and total intake assessment of vitamin D status

NHANES is a valuable source of information on vitamin D. Based on the previous IOM criteria, about $50 \%$ of adults, but fewer than one-third of older people, meet the recommended AI for vitamin D based on total intake. A new analysis is needed to assess what proportion of the population meets the currently recommended IOM intake criteria. Less than $10 \%$ of the US population have $25(\mathrm{OH}) \mathrm{D}$ levels that are generally considered deficient. The IOM evaluated the correlation of serum levels with inadequacy and the congruence of the data support $25(\mathrm{OH}) \mathrm{D}$ levels below $30 \mathrm{nmol} / \mathrm{L}$ with undesirable bone health outcomes (39); however, methodologic issues contribute uncertainty to this cutoff. The two ways to assess vitamin D status in the US population through intake and blood levels are not leading to the same conclusion. This is partially expected because sun exposure can be a significant contributor to vitamin D status and circulating $25(\mathrm{OH}) \mathrm{D}$ levels in addition to intake. These challenges in interpretation show how important it is to have access to both intake and biomarker data. Furthermore, the vitamin D case study shows the impact of methodologic issues, i.e. reformulations in laboratory methods, on the interpretation of trends in status over time.

\section{Conclusions}

The following is guidance on general requirements for monitoring nutrition status in large population studies and is derived from decades of NHANES experience.

1. The imprecision of the intake assessment method or biomarker assay should be low enough to enable detection of small trends. Due to the relatively large sample size of NHANES, small changes in population means can be easily detected. Fluctuations in the mean as a result of an imprecise assay can be mistaken for changes in population values.

2. The robustness of the intake assessment method or biomarker assay should be high enough to ensure comparability of data over time. As noted previously, fluctuations of the assay can be mistaken for changes in population values.

3. Development of a comprehensive and accurate food-composition database is key to assessment of dietary intake in the US population in NHANES. Lack of a complete and reliable database hindered dietary intake studies, including those needed to set dietary reference intakes.

4. Improvements in web-based software are needed to enable automated and self-administered 24-hr dietary recalls to be cost-effectively employed multiple times.

5. Use of one laboratory per test and preferably a central laboratory for the medium and high complexity testing helps to ensure consistent quality and comparability of data over time.

6. Use of kit biomarker assays for longitudinal studies may be problematic. To provide valid results, kit assays must be performed exactly as prescribed by the manufacturer, which rules out alteration of conditions to troubleshoot problems or improve the assay. Furthermore, re-calibration or refinement of the assay by the manufacturer cannot be ruled out and makes interpretation of long-term trends difficult.

7. Laboratory-developed biomarker tests are preferred, particularly when the measurement of the analyte has not yet been standardized, to allow in-house control over assay calibration and troubleshooting.

8. Whenever possible, using the same intake assessment method or biomarker assay is desirable to facilitate the comparability of data over time.

9. Often, changing procedures, either by choice (technology advances) or external stipulation (manufacturer phases out or reformulates assay) is unavoidable. A well-designed cross-over study is needed to enable data comparison over time.

10. Internal quality control, regular assay verifications, and external quality assurance are essential to ensure and document technical proficiency of the laboratory and the performance of the biomarker assay over time.

11. Reference materials should be used regularly, when available, to assess the bias of the biomarker assay and document consistent performance over time.

12. The traceability of the biomarker assay to a higherorder reference method is desirable because it is an important step to assay standardization and making laboratory data comparable. 
To effectively formulate and later evaluate nutrition policy, many data pieces are needed. The following list is by no means all inclusive:

1. Nutrition biomarker data - when possible, multiple biomarkers should be assessed to facilitate interpretation of the data.

2. Dietary intake data - data available should allow estimation of usual intakes to minimize intraindividual variation.

3. Supplement usage information - to allow a separate assessment of nutrient sources.

4. Demographic characteristics - to describe the nutritional status of the population by relevant subpopulations.

5. Behavioral factors - to explain differences seen in nutritional status.

6. Health indicators - to study associations between nutritional status and health.

7. Sample various population groups - to assess whether special groups of interest may have additional nutritional needs.

8. Monitoring over multiple years - to allow analysis of time trends.

Methods to assess nutrition status continually evolve; assays to measure biomarkers as well as dietary assessment methods are subject to constant change and improvement. As a result, a comprehensive qualityassessment system should be implemented at the onset of the survey to ensure meaningful data interpretation later. Utilization of combinations of biomarkers and dietary questionnaire information helps to better understand nutrition effects in relation to disease occurrence.

\section{Conflict of interest and funding}

The findings and conclusions in this report are those of the authors and do not necessarily represent the official views or positions of the Centers for Disease Control and Prevention/Agency for Toxic Substances and Disease Registry, the National Institutes of Health, or the Department of Health and Human Services. No grant/funding support is declared. No financial disclosure is declared.

\section{References}

1. CDC, NCHS, NHANES. 2011. Hyattsville, MD. http://www. cdc.gov/nchs/nhanes.htm [cited 1 March 2011].

2. Combs GF. Arnold Edward Schaefer (1917-1992). J Nutr 1993; 123: 2045-8.

3. CDC, NCEH. Nutrition report, 2008. Atlanta, GA. http:// www.cdc.gov/nutritionreport [cited 13 December 2010].

4. Thompson FE, Subar AF, Loria CM, Reedy JL, Baranowski T. Need for technological innovation in dietary assessment. J Am Diet Assoc 2010; 110: 48-51.
5. Subar AF, Thompson FE, Kipnis V, Midthune D, Hurwitz P, McNutt S, et al. Comparative validation of the Block, Willett, and National Cancer Institute Food Frequency Questionnaires. Am J Epidemiol 2001; 154: 1089-99.

6. The Nutrition Coordinating Center (NCC). University of Minnesota, 2009. http://www.ncc.umn.edu [cited 13 December 2010].

7. Raper N, Perloff B, Ingwersen L, Steinfeldt L, Anand J. An overview of USDA's dietary intake data system. J Food Compost Anal 2004; 17: 545-55.

8. Bailey RL, Mitchell DC, Miller C, Smiciklas-Wright H. Assessing the effect of underreporting energy intake on dietary patterns and weight status. J Am Diet Assoc 2007; 107: 64-71.

9. Dixon LB, Subar AF, Wideroff L, Thompson FE, Kahle LL, Potischman N. Carotenoid and tocopherol estimates from the $\mathrm{NCI}$ diet history questionnaire are valid compared with multiple recalls and serum biomarkers. J Nutr 2006; 136: 3054-61.

10. Signorello LB, Buchowski MS, Cai Q, Munro HM, Hargreaves MK, Blot WJ. Biochemical validation of food frequency questionnaire-estimated carotenoid, $\alpha$-tocopherol, and folate intakes among African Americans and non-Hispanic whites in the Southern Community Cohort Study. Am J Epidemiol 2010; 171: 488-97.

11. National Institutes of Health, National Cancer Institute (NCI). NHANES food frequency questionnaire, 2008. Bethesda, MD. http://riskfactor.cancer.gov/diet/usualintakes/ffq.html [cited 13 December 2010].

12. CDC, NCHS. NHANES, 2011. Hyattsville, MD. http:// www.cdc.gov/nchs/tutorials/Dietary/index.htm [cited 1 March 2011].

13. National Institutes of Health, National Cancer Institute (NCI). Usual dietary intakes, 2011. Bethesda, MD. http://riskfactor. cancer.gov/diet/usualintakes [cited 1 March 2011].

14. USDA. Agricultural research service. What we eat in America, 2010. Beltsville, MD. http://www.ars.usda.gov/foodsurvey [cited 13 December 2010].

15. USDA. Agricultural research service. Food and nutrient database for dietary studies, 2011. Beltsville, MD. http://www. ars.usda.gov/Services/docs.htm?docid=12089 [cited 1 March 2011].

16. USDA. Agricultural research service. National nutrient database for standard reference, release 23, 2011. Beltsville, MD. http://www.ars.usda.gov/Services/docs.htm?docid=8964 [cited 1 March 2011].

17. FDA. Food standards: amendment of standards of identity for enriched grain products to require addition of folic acid. Final rule. Fed Regist 1996; 61: 8781-97.

18. Feinleib M, Beresford SAA, Bowman BA, Mills JA, Rader JI, Selhub J, et al. Folate fortification for the prevention of birth defects: case study. Am J Epidemiol 2001; 154: S60-9.

19. IOM FNB. Dietary reference intakes: thiamin, riboflavin, niacin, vitamin B6, folate, vitamin B12, pantothenic acid, biotin, and choline. Washington, DC: National Academy Press; 1998.

20. McDowell MA, Lacher DA, Pfeiffer CM, Mulinare J, Picciano MF, Rader JI, et al. Blood folate levels: the latest NHANES results. NCHS data briefs, no 6. Hyattsville, MD: National Center for Health Statistics; 2008.

21. Wright JD, Bialostosky K, Gunter EW, Carroll MD, Najjar MF, Bowman BA, et al. Blood folate and vitamin B12: United States, 1988-94. Vital Health Stat 11 1998; 243: 1-78.

22. Pfeiffer CM, Osterloh JD, Kennedy-Stephenson J, Picciano MF, Yetley EA, Rader JI, et al. Trends in circulating concentrations of total homocysteine among US adolescents and adults: findings from the 1991-1994 and 1999-2004 National Health and Nutrition Examination Surveys. Clin Chem 2008; 54: 801-13. 
23. Jacques PF, Rosenberg IH, Rogers G, Selhub J, Bowman BA, Gunter EW, et al. Serum total homocysteine concentrations in adolescent and adult Americans: results from the third National Health and Nutrition Examination Survey. Am J Clin Nutr 1999; 69: 482-9.

24. Pfeiffer CM, Caudill SP, Gunter EW, Bowman BA, Jacques PF, Selhub J, et al. Analysis of factors influencing the comparison of homocysteine values between the third National Health and Nutrition Examination Survey (NHANES) and NHANES 1999+. J Nutr 2000; 130: 2850-4.

25. Yetley EA, Rader JI. The challenges of regulating health claims and food fortification. J Nutr 1996; 126: 765S-72S.

26. Wald NJ, Law MR, Morris JK, Wald DS. Quantifying the effect of folic acid. Lancet 2001; 358: 2069-73.

27. Pfeiffer CM, Johnson CL, Jain RB, Yetley EA, Picciano MF, Rader JI, et al. Trends in blood folate and vitamin B12 concentrations in the United States, 1988-2004. Am J Clin Nutr 2007; 86: 718-27.

28. Daly S, Mills JL, Molloy AM, Conley M, McPartlin J, Lee JL, et al. Low-dose folic acid lowers plasma homocysteine levels in women of child-bearing age. Q J Med 2002; 95: 733-40.

29. CDC. Recommendations for the use of folic acid to reduce the number of cases of spina bifida and other neural tube defects. Morb Mort Wkly Rep 1992; 41: 1-7.

30. Dietrich M, Brown CJP, Block G. The effect of folate fortification of cereal-grain products on blood folate status, dietary folate intake and dietary folate sources among adult nonsupplement users in the United States. J Am Coll Nutr 2005; 24: $266-74$

31. Yang Q-H, Carter HK, Mulinare J, Berry RJ, Friedman JM, Erickson JD. Race-ethnicity differences in folic acid intake in women of childbearing age in the United States after folic acid fortification: findings from the National Health and Nutrition Examination Survey, 2001-2002. Am J Clin Nutr 2007; 85: 1409-16.

32. Bailey RL, Dodd KW, Gahche JJ, Dwyer JT, McDowell MA, Yetley EA, et al. Total folate and folic acid intake from foods and dietary supplements in the United States: 2003-2006. Am J Clin Nutr 2010; 91: 231-7.
33. Yang Q, Cogswell ME, Hamner H, Carriquiry A, Bailey LB, Pfeiffer CM, et al. Folic acid source, usual intake, and folate and vitamin B12 status in US adults, 2003-2006. Am J Clin Nutr 2010; 91: 64-72.

34. Costello R. Vitamin D and health in the 21st century: federal initiatives to advance research. Am J Med Sci 2009; 338: 34-9.

35. IOM FNB. Dietary reference intakes: calcium, phosphorus, magnesium, vitamin D, and fluoride. Washington, DC: National Academy Press; 1997.

36. Holden JM, Lemar LE, Exler J. Vitamin D in foods: development of the US Department of Agriculture database. Am J Clin Nutr 2008; 87: 1092S-6S.

37. Yetley EA, Pfeiffer CM, Schleicher RL, Phinney KW, Lacher DA, Christakos S, et al. NHANES monitoring of serum 25hydroxyvitamin D - a roundtable summary. J Nutr 2010; 140: 2030S-45S.

38. Looker AC, Pfeiffer CM, Lacher DA, Schleicher RL, Picciano MF, Yetley EA. Serum 25-hydroxyvitamin D status of the US population: 1988-1994 compared with 2000-2004. Am J Clin Nutr 2008; 88: 1519-27.

39. Yetley EA. Assessing the vitamin D status of the US population. Am J Clin Nutr 2008; 88: 558S-64S.

40. IOM. Dietary reference intakes for vitamin D and calcium. Washington, DC: National Academies Press; 2011. http://www. iom.edu/Reports/2010/Dietary-Reference-Intakes-for-Calciumand-Vitamin-D.aspx [cited 1 March 2011].

41. Bailey RL, Dodd KW, Goldman JA, Gahche JJ, Dwyer JT, Moshfegh AJ, et al. Estimation of total usual calcium and vitamin D intake in the United States. J Nutr 2010; 140: 817-22.

\footnotetext{
*Christine M. Pfeiffer

Centers for Disease Control and Prevention

4770 Buford Highway MS F55

Chamblee, GA 3034I, USA

Tel: + (770) 4887926

Fax: $+(770) 4884139$

Email: cpfeiffer@cdc.gov
} 